\title{
Teaching medical function experiments reformation and laboratory construction
}

\author{
Yu LI \\ Qiqihar Medical University \\ line 2: name of organization, acronyms acceptable \\ Qiqihar, Heilongjiang, China 161000
}

\begin{abstract}
Medical function experimental teaching platform is an important foundation to support the teaching of medical experiments; it's significant in cultivating students' creative ability. Seeing from the three aspects of pilot project, open experimental teaching platform, digitized virtual experiment platform, making a discussion about building a new function experiment teaching platform. Construction of the new function experiment teaching platform make limited experimental resources been fully utilized, in order to enhance the effectiveness of experimental teaching and training of qualified medical personnel.
\end{abstract}

Keywords- functional experiments; experimental teaching; laboratory construction

\section{INTRODUCTION}

Teaching medical function experiments mainly includes physiology, pharmacology, and path physiology three experimental courses. Previous experiment teaching modes of these subjects are belonging to each department, theoretical and experimental teaching are simultaneously, the experiment is limited to the discipline. And our school follows the teaching reformation, from 1992, the physiology, path physiology; pharmacology began reconstruction, set up a medical laboratory functional test center based on technicians. Center opened an independent discipline medical function experiment. The establishment of the center have such benefits: the regulation of various disciplines of construction and development on the macro; sharing resources, improving the utilization of equipment; and strengthening horizontal linkages between disciplines, improve the overall quality of students; improving the teaching level and in favor of improving the quality of teachers.

\section{THE STATUS AND ROLE OF FUNCTION EXPERIMENT TEACHING IN MEDICAL RESEARCH, PROFESSIONAL AND TECHNICAL EDUCATION}

Medical research has made significant discoveries and achievements, and it needs to use a variety of experimental research techniques, methods and theories of knowledge, and integrated means, to a certain extent, it depends on the establishment of new thinking and new methods of experimental techniques. Such as the establishment and development of medical molecular biology, cell biology, genetic engineering and other experimental techniques, it has solved many difficult problems in diagnosis and treatment of diseases.

Basic medical disciplines are established and developed on the basis of experimental studies on the functional experiment technique is a method and means that is widely used in medical science, is an important condition for the foundation of modern medical science and technology development.

\section{MEDICAL FUNCTION EXPERIMENT TEACHING REFORM} MEASURES

\section{A. change teaching concepts.}

With the development of science and technology, society needs more high-quality, complex talent, which is a higher medical education requirement and new meaning. The traditional knowledge-based traditional medicine experimental teaching model can not meet the requirements of modern science and technology personnel training, training medical students' comprehensive ability and creativity has become an important goal of medical education. Therefore, teachers teach students not only to give ready-made knowledge, but also to guide students to explore the realm of the unknown, the main role of teachers is to guide students to reflect on effective learning, rather than simply impart knowledge to students. In the experiment teaching, teachers should change teaching ideas, adhere to the principle of quality education based on creative thinking and innovation, establishing the concept that students are learning subject, stimulate their desire for knowledge, to teach students to master the learning methods and research skills, guide students to scientific thinking, cultivate their ability to develop their practice with a grasp of scientific knowledge, methods and technology to create new knowledge and discovery, open innovation and technology.

\section{B. Adjust the teaching content and methods.}

Medical science is not a simple patchwork of experimental physiology, path physiology and pharmacology subject experiments, but the combination of the three experiments organic disciplines. Past Functional Experimental Teaching culture experiments focus on verify the theory and skills-based, and in the future it will emphasis on research and experimental culture, innovation. To achieve 
this goal, we follow the reduction of validation experiments, increasing the synthesis and design principles of the experiment; redesign the contents of the original experimental physiology, path physiology and pharmacology, scientific arrangement, a combination of medical function experiments tutorials, functional experimental content and requirements, to prepare the corresponding function experiment syllabus. Focus on students' comprehensive analysis of problem-solving skills during the experimental teaching, scientific thinking and rigorous scientific attitude training, the quality of education, innovation and education, capacity-building and experimental teaching in all aspects.

Follow the progressive approach in the teaching process, the law of step by step, the first is to introduce students to the basic techniques of animal experiments, such as the specification using common instruments and surgical instruments, how to hold experiment animals, anesthetized animals fixed method, basic knowledge about laboratory animals dose calculations, make students basic skills and functional experimental learning and training becomes systematic, standardized, and lay a good foundation for future experimental studies. Secondly, do the basis experimental verification of confirmatory experiments while streamlining the three original experimental science lessons, retains some representative classic experiments that enable to make students deepen and consolidate the relevant theoretical knowledge to understand and remember through experiments.

Comprehensive experiment is a test that a class needs to use the knowledge to complete; it contains three management expertises, content coherent and systematic. Students from the experiment observe the body from normal to pathological changes in physiological state and the entire process of drug treatment, through the experimental principle, experimental results of the analysis, the three classes of knowledge intersect each other, mutual penetration, and mastery. Because comprehensive experiment involving a wide range experiments and the experiment is difficult, the prep course should be offered before the experiment related content based on experimental arrangements, including experimental purposes, principles, methods, procedures, equipment, pharmaceuticals and related theories, experiments enable students to understand the whole process, strict standard operation, careful observation and accurate recording results. Comprehensive experiments enable students to develop a systematic understanding of disease processes, specifically the role of drug therapy, application of knowledge to improve the students' comprehensive ability to analyze and solve problems.

Carry out experiments is designed to develop and enhance students' scientific thinking, innovation and comprehensive research quality. Preliminary research by simulating the research process, make title, experimental design, training in writing papers, consciously develop their scientific awareness and scientific interest, so that students are familiar with the basic process of systematic and scientific way of obtaining scientific knowledge. Students are encouraged to continue to explore, to make them swim in the ocean of knowledge, to search, to efficiently do time tracking about the forefront technological development, learning new ways to discover new problems, to lay the foundation for future scientific research work. Before designing experiments, teachers must first explain to the students the basic principles and requirements of the experimental design, such as the three elements of the processing factor such as dealing factors, experimental design study, the experimental effect, the three principles of randomized, controlled, principle are repeat randomly, in the meantime introduce students the ways to view the collections and the use of Internet search tools such as literature search and obtain documentation through selection of statistical methods of data relevant knowledge. The whole operation process of designing experiments roughly divided into three steps, namely the topic and opening report, experimental operation and results analysis, concluding report. The main topics are self-determined by the students, the students use their spare time to search content they are interested in the related literature review, proposed experiments with certain innovative idea based on existing knowledge. After the panel discussion, each group selected a better design, by opening report in the form of design discussion, mainly on science, innovation, and to demonstrate the feasibility of the design scheme. Through discussions it can broaden students' knowledge, change their usual mode of thinking straight, so they can gradually learn divergent thinking. After the end of the discussion, each group forming a formal experiment embodiment according to the results of the discussion, then a formal experiment. Experimental operations include preparation by the students before the experiment independently, emphasizing teamwork spirit among the group operation, encountered problems will be helped by teachers to solve. After the end of the experiment, the students organize information, process medical statistical methods with experimental data, realistic analyze the experimental results, and use reference format to write a research paper essay test, the contents of the paper will be discussed in the form of concluding final report. By this stage of training, it will enable students to write papers and answer process and have a basic understanding. Throughout the design of the experimental teaching process, in order to fully reflect the student, it will enable students to do with fully autonomous, such as topics, test operations, data processing, thesis writing, and production and other slides as independently as possible. In addition, allowing students to fail in the experiment, it is important that the reason for the failure can be analyzed objectively and comprehensively summarizes the experience, and this is another sense of success.

\section{Laboratory.}

Laboratory is to create an environment conducive to innovation ability of the academic environment, to provide students with a good experimental environment for students to complete the design of the experiment is the basic guarantee. In the design of experimental stage, the laboratory is open to students throughout the day; students can choose according to their own protocols experimental time, ready to enter the lab to complete their pilot project. In the open 
laboratory, we must promptly develop and update the lab rules and regulations, to make it scientifically and standardized to ensure the normal operation of the laboratory, smooth track, more effective for the practice of teaching.

\section{Establish effective experimental evaluation mode.}

How to test assessment, to evaluate students' skills and level of real experiments, mobilize students to participate in the experimental teaching initiative is another core issue of Experimental Teaching Reform. As for the new function for experimental teaching model, we take the process of evaluate the results of combination, self-evaluation and evaluation of others, a combination of objective assessment of students. Experimental evaluation mainly from three aspects: First, the theory test, checking students' grasp of the theory of experimental methods; the second is skills assessment test, to check students' hands-on ability; the third is experimental design assessment, teachers based on student test design level, to test the actual ability, analytical ability and paper result writing skills comprehensive assessment, comprehensive evaluate students' self-learning ability, analyzing ability, problems solving and creative thinking skills.

\section{E. Improve teachers' own quality.}

Improve the overall quality of experimental teaching staff; the establishment of high-level, comprehensive technology experimental teachers is an important part of the experimental teaching reform. Teachers are the implementers of quality education, only highly qualified teachers can carry out high-level, high-quality experimental teaching job in order to cultivate high-quality personnel. The implement of designing of experiments on teachers put forward higher requirements, teachers need to have a superb and comprehensive experimental skills and strong research capabilities. Only widely read literature instructor, expand knowledge, understand the dynamic research, can instructors help students to determine good experimental design; in addition, in the implementation process of the experiment, only teacher with excellent experimental skills and the ability to rule out equipment failure before it can better solute the various problems and difficulties encountered in the experimental school students, reduce blindness due to students' lack of experience and reduce unnecessary experiments' loss. Therefore, teachers in their work, not only to focus on the rich theoretical knowledge, but also focus on experimental skills and scientific research continue to increase.

\section{ESTABLISH FUNCTION TEACHING EXPERIMENT PLATFORM}

\section{A. The intensive management of laboratory}

The development of higher medical education is closely related to the modernization of the laboratory. During the National Experimental Teaching Demonstration Center construction unit, basic medical experimental teaching center has always adhered to assessment and reform, evaluation and construction combined, focusing on construction principle. Focus on laboratory construction; strengthen scientific, modernization and standardization of laboratory management. Reforming Laboratory Management System, based on the sharing of resources, strengthen the connotation construction, and build a reasonable structure, operation and efficient management of the new laboratory system. Schools should increase investment in the construction of the experimental center, each year to update the configuration of high-tech laboratory equipment, thereby enhancing the lab. Establish and improve a variety of practical laboratory regulations, code of conduct scientific management. And the implementation of personal responsibility, law has rulebased. Strengthening the institutional laboratory equipment, and standardized management; the use of modern management tools to teaching computer file management.

\section{B. Construct high-efficiency integrated laboratory professional basis}

According to my school research university development criteria, to achieve functional experiments sub-centers overall planning; resource sharing optimizes the allocation of intensive management direction. Our experimental system established by eight professional basic comprehensive laboratories. It not only increases the level of teaching experiments, teaching and maximizes resource utilization and reduces resource waste and duplication. Achieve computerized laboratory automation.

Functional experimental class curriculum system performance discipline group established experimental teaching reform, highlighting functional experimental systemic and integrated to enhance students' practical ability and innovation. Laboratory changes from single, reproducibility into a professional comprehensive and costeffective shared paradigm shift, in order to deepen the reform of function experiment teaching materials to provide a solid guarantee.

\section{CONCLUSIONS}

In short, the medical function experiments reform is to adapt to the changing patterns of modern medical education; all aspects need to be further improved. In the future, we will continue to sum up the experience of teaching practice, forge ahead, and we will function continuously push forward the reform of experimental teaching.

\section{References}

[1] Hongqi Xiong, Yurong Dai, Zheng Jiamao, research and practice of experimental teaching reform and laboratory construction planning $[\mathrm{J}]$ Experimental Technology and Management, 2008,10: 1-4 + 8 .

[2] Liu Jie, Wang Qiujing, Liu Fen, Chen Li, Jing Li, medical function experiment teaching reform and laboratory construction $[\mathrm{J}]$ China Higher Medical Education, 2009,08: 116-117.

[3] Zhao Weihong, Zhao Weiping, medical practice and exploration of function experiment teaching reform [J] China Higher Medical Education, 2005,06: 45-46.

[4] Deng Fengmei, He Fang, Sun Zhiping, Liu Mei, Chucheng Jing, Zhong Hua medical function experiments Teaching Practice and Discussion [J] Land Reclamation Medicine, 2004,01: 48-49. 
[5] Gao Zhiping Interferon - Huang Honglin, Hu Bute, Tang Chaoke, medical function experiments reform in development thinking [J] Medicine and Society, 2004,05: 63-65.

[6] Gao Zhiping, Li Bo Ping, Tang Chaoke, Huang Honglin Hu Bute, Liao Duanfang, - medical function experiments Teaching Quality Education [J] Northwest Medical Education, 2004,02: 135-137.

[7] Li Yan, Yin Lei, Liang Yueqin medical function experiment teaching reform and practice [J] basic medical education, 2011,11: 1011-1012.
[8] Zhang Minghao, Yu-Jie Li, Sun Shuguang, Pan Xiaoli, Han Qianqian, Li Yaping thinking about new medical function experiment teaching platform construction [J] Chinese Medicine, 2015,02: 113-116 + 124 . 